
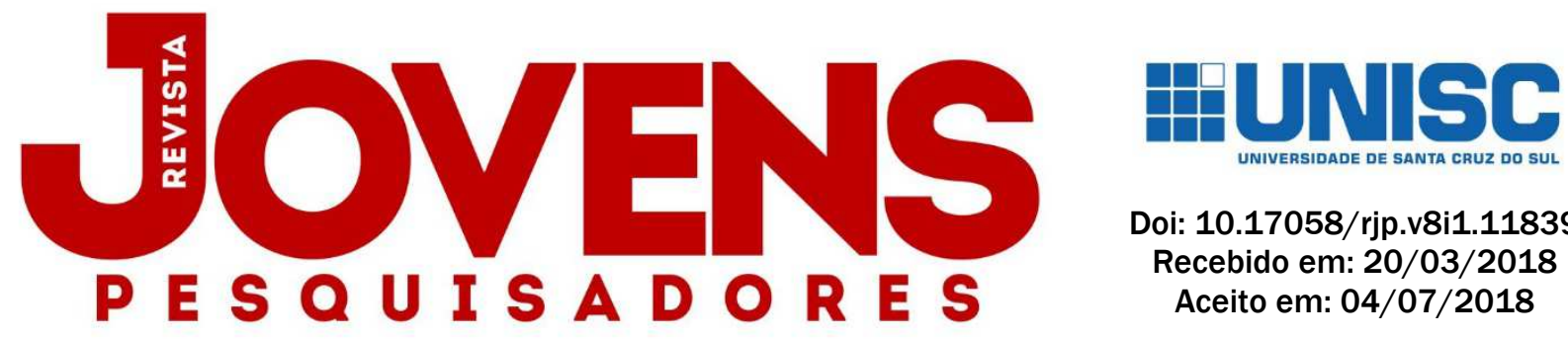

Doi: $10.17058 /$ rjp.v8i1.11839

Recebido em: 20/03/2018

Aceito em: 04/07/2018

\title{
PENSANDO SOBRE A POPULAÇÃO IDOSA RURAL DE SANTA CRUZ DO SUL
}

\author{
ROHDE, J.1; AREOSA, S. V. C. ${ }^{2}$ \\ PALAVRAS CHAVE: População idosa. Meio rural. Migração.
}

\begin{abstract}
RESUMO
Neste recorte de um projeto de pesquisa intitulado "Estudo Socioeconômico e Demográfico da População Idosa no Meio Rural do Município de Santa Cruz do Sul”, de caráter quantitativo, serão apresentadas características do envelhecimento no meio rural de Santa Cruz do Sul, através de dados secundários, provenientes do Censo Demográfico da População de 2010 (IBGE, 2010), bem como resultados preliminares dos dados primários, cuja amostra é equivalente a $10 \%$ do total de idosos dos sete distritos rurais do município. A pesquisa evidencia que há uma redução da população rural jovem, que migra para os centros urbanos em busca de melhores condições de vida. Esta percepção se dá mediante a realidade encontrada pelas autoras nos distritos, pois um número expressivo de idosos vive apenas com o cônjuge ou mesmo sozinho. A partir deste olhar sobre a população idosa, dá-se visibilidade para a sua realidade, bem como para as diferenças entre viver a velhice no campo e na cidade.
\end{abstract}

\section{THINKING ABOUT THE ELDERLY RURAL POPULATION OF SANTA CRUZ DO SUL}

KEYWORDS: Elderly population. Countryside. Migration.

\begin{abstract}
In this research project "Socioeconomic and Demographic Study of the Elderly Population in the Rural Environment of the Municipality of Santa Cruz do Sul", quantitative characterization, characteristics of aging in the rural environment of Santa Cruz do Sul will be presented through secondary data from the Population Demographic Census of 2010 (IBGE, 2010), as well as preliminary results of the primary data, whose sample is equivalent to $10 \%$ of the total number of elderly of the seven rural districts of the municipality. The research shows that there is a reduction of the young rural population, which migrates to the urban centers in search of better living conditions. This perception through the reality found by the authors in the districts, since an expressive number of elderly people lives only with the spouse or even alone. From this contact and knowledge of the elderly population, visibility is given to their reality, as well as to the differences between living old age in the countryside and in the city.
\end{abstract}

\footnotetext{
1 Graduada em Psicologia pela Universidade de Santa Cruz do Sul.

2 Docente do Departamento de Psicologia na Universidade de Santa Cruz do Sul.
} 


\section{INTRODUÇÃO}

Há muitas diferenças entre o meio urbano e o meio rural. Envelhecer nestes dois espaços, portanto, também tem diferenças e particularidades. As diferenças entre estes espaços iniciam na dimensão populacional e geográfica, mas vão muito além, como o acesso a serviços públicos, de saúde, transporte, assistência; atividades de lazer, costumes, crenças e representações sociais, o que torna o processo de envelhecer no meio rural muito particular. (BERTUZZI; PASKULIN; MORAIS, 2012).

Há algumas outras características que evidenciam vulnerabilidades no meio rural, em que, de forma geral, costuma-se encontrar altos índices de pobreza da população, baixo nível no ensino e educação, moradias precárias, dificuldades para locomoção e transporte, altas taxas de problemas de saúde e dependência. (BERTUZZI; PASKULIN; MORAIS, 2012). Misso e Portella (2003) referem que o meio rural estaria para o meio urbano como uma espécie de "periferia", dependendo dela política, econômica e socialmente.

Alcântara (2016) refere que ao estudar o envelhecimento no contexto brasileiro, torna-se importante conhecer mais sobre o espaço geográfico desta população, ou seja, onde estão, e onde vivem estes idosos. 0 difícil acesso às localidades rurais, menos disponibilidade de recursos já referidos, e precariedade de políticas públicas enfatizam a necessidade de pesquisas neste espaço.

Os idosos que vivem no meio rural geralmente enfrentam mais dificuldades se comparados aos que vivem no meio urbano, como menor oferta e maior dificuldade de acesso aos serviços citados anteriormente, e que são considerados de suma importância para uma boa qualidade de vida. Na busca por tentar compreender algumas destas questões, o projeto de pesquisa "Estudo Socioeconômico e Demográfico da População Idosa no Meio Rural do Município de Santa Cruz do Sul", que é realizado pela Universidade de Santa Cruz do Sul através de financiamento captado junto ao Conselho Municipal do Idoso de Santa Cruz do Sul, se propõe a desvelar a realidade em que vivem os idosos no meio rural do município.

O estudo também busca verificar as condições econômicas e sociodemográficas destes idosos, investigando suas representações sociais sobre a velhice e traços culturais que marcam suas trajetórias de vida. Este artigo tem por objetivo apresentar algumas características do envelhecimento no meio rural de Santa Cruz do Sul, chamando atenção para o tema. A seguir se apresentará a metodologia do estudo, seguida por alguns resultados preliminares já analisados e uma discussão com autores que debatem a área, após as considerações mais relevantes. 


\section{METODOLOGIA}

A pesquisa realizada trata-se de um estudo descritivo, do tipo exploratório. Apresenta dados secundários, provenientes do Instituto Brasileiro de Geografia e Estatística (IBGE), assim como dados primários, coletados nos sete distritos participantes da pesquisa mediante aplicação de questionário.

Os dados secundários são provenientes do Censo Demográfico da População de 2010 (IBGE, 2010). Já os dados primários foram coletados em 2017 através da aplicação de questionários estruturados para conhecer o perfil sociodemográfico da população idosa que vive no meio rural do município de Santa Cruz do Sul/RS. A amostra é do tipo espontânea e equivale a 10\% do total de idosos em cada localidade, ou seja, sete distritos rurais do município: Alto Paredão; Saraiva; São Martinho; Rio Pardinho; Boa Vista; Monte Alverne e São José da Reserva. A amostra foi composta por mulheres e homens com idades que variaram entre 60 e 92 anos, totalizando 232 pessoas.

Os questionários foram aplicados pelo grupo de pesquisa em Unidades Básicas de Saúde, Estratégias de Saúde da Família (ESF), Hospital, grupos de convivências e em residências, por agentes comunitários nas localidades mais distantes. Os idosos que concordaram em participar da pesquisa assinaram o termo de consentimento livre e esclarecido dando o seu assentimento. Para realizar a análise dos resultados dos questionários aplicados, as respostas obtidas foram organizadas em tabelas no Microsoft Excel, para posterior análise de dados no StatisticalPackage for the Social Sciences (SPSS).

\section{O ENVELHECIMENTO NO MEIO RURAL}

Assim como grande parte dos países do mundo, o Brasil também vem apresentando mudanças em sua pirâmide etária. Em 1980, a população idosa brasileira era composta por 7,2 milhões de pessoas, o que representava cerca de $6,1 \%$ do total da população. (CAMARANO; KANSO; FERNANDES, 2016).

O Censo de 2010 definiu a estrutura brasileira por sexo e idade, e levantou inúmeras informações que permitem conhecer os padrões de fecundidade, mortalidade e migração (interna e externa) e projetar estimativas sobre as transformações demográficas em curso. Estas informações são a base para pensarmos as necessidades de políticas das áreas de saúde, previdência e educação da população em geral e de assistência a grupos específicos mais vulneráveis como as crianças, os adolescentes e os idosos. (IBGE, 2010). Conforme dados do último censo demográfico, o número de idosos no país passou para 20,6 milhões, significando cerca de $11 \%$ da população total. Calcula-se que em 2025 haja em média mais de 800 milhões de pessoas com idade superior a 65 anos em todo o mundo. (FECHINE; TROMPIERI, 2012). 
As elevadas taxas de fecundidade havidas nas décadas de 1950 e 60, seguidas pela redução do número de filhos e da diminuição da mortalidade em todas as faixas etárias no Brasil desde os anos 1950, explicam esta nova configuração da população do país. Isso significa que os idosos de hoje têm vivenciado uma época em que a qualidade de vida foi e está sendo melhorada, contribuindo para o aumento da expectativa de vida. (CAMARANO; KANSO; FERNANDES, 2016).

As informações de natureza demográfica obtidas pelo IBGE, que faz a cada 10 anos o censo populacional, são primordiais para a elaboração das projeções e estimativas, permitindo analisar a evolução da população do país nos diferentes aspectos como etnia, religião, migração, renda, etc. Além disso, estes dados populacionais servem de parâmetro para distribuição de verbas federais aos fundos estaduais e municipais, bem como, para definir o número dos representantes dos cidadãos nas assembleias legislativas municipais. Segundo o IBGE, o Censo de 2010, segue as recomendações internacionais, o que torna possível comparar o País com diferentes regiões do mundo em muitos aspectos no que tange ao desenvolvimento social e econômico, sendo um avanço para a realização de pesquisas secundárias com a utilização de seus dados processados.

No Vale do Rio Pardo, segundo dados secundários do IBGE do ano de 2010, verificamos que a região possuía uma população de 418.141 habitantes e, desse total, 60.120 eram idosos, o que significa $14,37 \%$ da população total. Houve um crescimento da população idosa de $2,89 \%$ entre o censo de 2000 para o de 2010. Esses dados demonstram um significativo envelhecimento da população na região do Vale do Rio Pardo, que tem índices maiores do que o do estado do Rio Grande do Sul que, em 2010, tinha uma população total de 10.693 .929 habitantes. Destes, 1.459.597 eram idosos, que representavam 13,65\% da população. Em termos de Brasil, a região também se destaca pois, na última contagem populacional, o país tinha 190.755.799 habitantes, com um número total de idosos de 20.590 .597 , o que representava que $11 \%$ da população do país tinha mais de 60 anos.

Assim, a partir dos dados secundários do IBGE, conseguimos olhar para o município em estudo e verificar a sua realidade no ano de 2010. Em Santa Cruz do Sul, havia 15.559 idosos (13,14\% da população), na última contagem populacional.Quanto à zona rural do município, a população idosa era a seguinte:

Tabela 1 -População idosa rural de Santa Cruz do Sul

\begin{tabular}{c|c|c}
\hline Distrito & $\begin{array}{c}\text { População } \\
\text { idosa }\end{array}$ & $\begin{array}{c}\text { \% de idosos em relação à pop. } \\
\text { total }\end{array}$ \\
\hline Alto Paredão & 219 & $13,29 \%$ \\
\hline Boa Vista & 416 & $19,63 \%$ \\
\hline Monte Alverne & 574 & $20,55 \%$ \\
\hline Rio Pardinho & 532 & $20,30 \%$ \\
\hline São José da Reserva & 107 & $15 \%$ \\
\hline São Martinho & 140 & $39,77 \%$ \\
\hline Saraiva & 196 & $19,83 \%$ \\
\hline Total & 2.184 & $14,04 \%$
\end{tabular}

Fonte: IBGE, 2010. 
Este alto percentual de idosos na zona rural, com índices mais elevados de idosos sobre o total da população do que os percentuais encontrados sobre a população urbana, está em consonância com o que nos diz Sakamoto (2014): há uma redução da população rural jovem, que migra para os centros urbanos em busca de melhores condições de estudo e de trabalho, na perspectiva de melhora de vida.

Sakamoto e Maia (2013) entendem que há um incentivo da vida urbana, gerando uma espécie de abandono do meio rural, principalmente pelos jovens. Tal realidade provoca a redução dos membros da família, da força de trabalho nas propriedades, e da possibilidade de que eles exerçam o cuidado com os mais velhos.

\begin{abstract}
Apesar de avanços importantes no que se refere aos ganhos de produtividade e rendimento das ocupações agrícolas, é nas áreas urbanas que se concentram as ocupações mais promissoras no que se refere ao destino socioeconômico das pessoas, seja pela perspectiva de estabilidade econômica, seja pelo próprio prestígio social das ocupações. (QUADROS; MAIA, 2010. p. 445).
\end{abstract}

As projeções do IBGE (2010), bem como a análise de autores como Carvalho e Wong (2008) preveem que, entre 2000 e 2050, 88 milhões de pessoas serão, provavelmente, adicionadas à população total.

Porém alertam para o fato de que, entre os jovens, as taxas de crescimento negativas prevalecerão, o que trará, como consequência, a diminuição no número absoluto deste segmento populacional. Durante o período referido, o tamanho da população com menos de 15 anos deverá decrescer em algo próximo de cinco milhões. Enquanto, entre 2000 e 2050, a população total deverá crescer cerca de 50\%, aquela abaixo de 15 anos declinaria em torno de $10 \%$.

Figura 1 - Razões de dependência (RD) e quociente de idosos-jovens (QIJ). Brasil, 2000-2050.

\begin{tabular}{|c|c|c|c|c|c|c|c|}
\hline \multirow[t]{3}{*}{ Periodo } & \multicolumn{6}{|c|}{ RD } & \multirow[t]{3}{*}{ Qlग $(x 100)$} \\
\hline & \multicolumn{3}{|c|}{ Razāo (x 100) } & \multicolumn{3}{|c|}{ Distribuição relativa (\%) } & \\
\hline & Total * & Jovem ** & Idosa $* \star \star$ & Total & Jovem & Idosa & \\
\hline 1950 & 80,3 & 74,9 & 5,4 & 100,0 & 93,3 & 6,7 & 7,2 \\
\hline 1975 & 79,2 & 72,2 & 7,0 & 100,0 & 91,2 & 8,8 & 9,6 \\
\hline 2000 & 54,3 & 45,9 & 8,4 & 100,0 & 84,6 & 15,4 & 18,3 \\
\hline 2025 & 48,7 & 33,4 & 15,3 & 100,0 & 68,6 & 31,4 & 45,7 \\
\hline 2050 & 57,8 & 28,1 & 29,7 & 100,0 & 48,6 & 51,4 & 105,6 \\
\hline 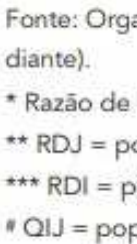 & $\begin{array}{l}\text { das Naçō } \\
\text { dência Tot. } \\
\text { o menor d } \\
\text { o de } 65 \text { a } \\
\text { com } 65 \text { ar }\end{array}$ & $\begin{array}{l}\text { Unidas ' (até } 1 \\
\text { RDT) = Razāo } \\
5 \text { anos/popul } \\
\text { ou mais/pop } \\
\text { ou mais/popa }\end{array}$ & $\begin{array}{l}\text { 5, dados bru } \\
\text { Dependênc } \\
\text { o de 15-64; } \\
\text { çáo de 15-6 } \\
\text { ão com me }\end{array}$ & $\begin{array}{l}\text { Institutc } \\
\text { Jovens }\end{array}$ & $\begin{array}{l}\text { iro de Ge } \\
\text { Razāo de }\end{array}$ & e Estati & $\begin{array}{l}2 \text { (2000 em } \\
\text { Idosos (RDI): }\end{array}$ \\
\hline
\end{tabular}

Fonte: Carvalho; Wong, 2008, p. 601. 
Se observarmos as projeções da figura 1, entre 1950 e 2050, a população idosa sairia de um percentual de em torno de 5\% da população total para quase 30\%. Já a tendência dos jovens é uma diminuição de cerca de $75 \%$ que representavam sobre a população total em 1950 para $28 \%$ em 2050, ou seja, uma inversão na pirâmide etária.

Este fenômeno também se evidencia nạ realidade encontrada pelas autoras na pesquisa atual, pois nos sete distritos estudados, um número expressivo de idosos vive apenas com o cônjuge ou mesmo sozinho. Neste contexto, torna-se importante olhar para o fato de que vem diminuindo a disponibilidade de cuidadores familiares, uma vez que os jovens estão migrando para as cidades.

0 processo de envelhecimento não está associado à doença, no entanto é uma fase da vida onde perdas funcionais e cognitivas são esperadas, o que implica em necessidade de auxílio e apoio para realização de algumas tarefas. Portanto, os idosos que acabam ficando sozinhos no campo, sobrecarregam-se com o trabalho rural e com suas atividades da vida cotidiana, bem como é gerado um sentimento de solidão e isolamento. (BERTUZZI; PASKULIN; MORAIS, 2012).

A tabela 2 apresenta os dados que ressaltam tais aspectos.

Tabela 2 - Idosos que vivem sozinhos ou apenas com cônjuge

\begin{tabular}{c|c|c|c}
\hline Distrito & N de idosos entrevistados & $\begin{array}{c}\text { Vive } \\
\text { sozinho(a) }\end{array}$ & Vive com o Cõnjuge \\
\hline $\begin{array}{c}\text { São José da } \\
\text { Reserva }\end{array}$ & 12 & 3 & 6 \\
\hline Alto Paredão & 23 & 5 & 15 \\
\hline Boa Vista & 43 & 3 & 16 \\
\hline Saraiva & 24 & 5 & 18 \\
\hline Monte Alverne & 55 & 5 & 34 \\
\hline Rio Pardinho & 52 & 0 & 13 \\
\hline São Martinho & 19 & 24 & 138 \\
\hline Total & 228 & 3 & \\
\hline
\end{tabular}

Fonte: coleta de dados da pesquisa, 2017.

A tabela 2 nos permite observar que, da amostra pesquisada nos distritos de Santa Cruz do Sul, temos 24 idosos que vivem sozinhos e 138 que vivem com o cônjuge ou companheiro, ou seja, mais da metade dentre o total de entrevistados vive em condição marital.

Acredita-se que vários fatores podem estar contribuindo com este fenômeno, como a queda da fecundidade, a fragmentação das famílias, a modernização da agricultura e as extremas desigualdades territoriais. Froehlichet al. (2011) falam do intenso e homogêneo êxodo rural até a década de 1980, uma vez que famílias inteiras migravam para o meio urbano. Nas últimas décadas 
este processo se modificou, e hoje os que mais migram para as cidades são os jovens, de modo que acabam ficando mais idosos no espaço rural.

Também há a questão da aposentadoria rural, política pública que garante a concessão da aposentadoria aos 55 anos para as mulheres e aos 60 anos para os homens que trabalharam no campo. Esta renda tem contribuído para a permanência dos idosos neste território. (SAKAMOTO, 2013).

Tavares et al. (2011) falam da importância da aposentadoria rural para os idosos que vivem nestes espaços, referenciando que ela tem papel muito significativo no orçamento familiar. A aposentadoria, apesar de em muitos casos não conseguir, sozinha, garantir ao idoso do campo todo o seu sustento e provimento das necessidades, representa uma segurança diante dos tempos difíceis, de que o alimento não irá faltar. Portanto, é sinônimo de dignidade. (ALCÂNTARA, 2016).

A aposentadoria rural tem contribuído para a elevação da renda no campo, bem como para a erradicação da pobreza, desempenhando um importante papel social. (BELTRÃo et al. 2004). Missio e Portela (2003, p. 34) falam da melhora na qualidade de vida dos beneficiários, oportunizando muitas vezes que o idoso desfrute da sua vida, explicando que, para quem não tem renda mensal fixa, o que é muito comum nas áreas rurais, a aposentadoria "traz o status de independência". Desta forma a contribuição da aposentadoria rural pode ser um fator que esteja mantendo esses idosos em suas propriedades no campo.

A permanência dos idosos no meio rural se deve a várias questões, como o êxodo rural, o incentivo da vida urbana, a busca por melhores condições de vida por parte dos jovens costumeiramente vislumbrada nas cidades - e a aposentadoria rural como um dispositivo que permite a permanência do idoso no campo. Tais particularidades do envelhecimento desta população sugerem a importância de olhar para este segmento populacional que ainda é pouco visado e assistido, que pouco consegue acessar alguns recursos, seja por distância, dificuldade de transporte, condições de saúde ou outras questões.

\section{CONSIDERAÇÕES FINAIS}

O envelhecimento é, como já referido anteriormente, um fenômeno global, e no meio rural de Santa Cruz do Sul também está evidente, com distritos onde mais de $20 \%$ da população é composta por pessoas idosas. Destes idosos que vivem em zona rural, um percentual significativo vive sozinho ou com o cônjuge que, em sua grande maioria, também é idoso. Ou seja, os idosos estão ficando sozinhos no campo.

Dentro desta perspectiva, faz-se importante atentar também para o fato de que quanto mais sozinhos no campo ficam os idosos, menor é sua rede de suporte social e de cuidados, o que dificulta ainda mais um processo de envelhecimento digno e saudável. 
A partir deste contato e conhecimento da população idosa, dá-se visibilidade para a sua realidade, bem como para as diferenças entre viver a velhice no campo e na cidade. Desta forma, é possível pensar em planejamento e estratégias para lidar com as especificidades e possíveis dificuldades que se apresentam ao envelhecimento com qualidade de vida no meio rural. Estas são algumas das grandes preocupações que se defrontam na atualidade os Estados e as comunidades científicas quando a temática a ser debatida é o fenômeno do envelhecimento demográfico.

\section{REFERÊNCIAS}

ALCÂNTARA, Adriana. Envelhecer no contexto rural: a vida depois do aposento. In: ALCÂNTARA, A.; CAMARANO, A.A; GIACOMIN, K.C. (Org). Políticas Nacional do Idoso: velhas e novas questões. Rio de Janeiro: IPEA, 2016, p. 323-342.

BELTRÃO, Kaizôet al. A Constituição de 1988 e o Acesso da População Rural Brasileira à Seguridade Social. In: CAMARANO, Ana Amélia (org.). Os novos idosos brasileiros muito além dos 60? Rio de Janeiro: IPEA, 2004, p. 321-351.

BERTUZZI, D; PASKULIN, L.G.M; MORAES, E.P. Arranjos e Rede de Apoio Familiar de Idosos que vivem em uma Área Rural. Texto \& Contexto Enfermagem, v. 21, n.1, p. 158-166 Florianópolis Jan./Mar. 2012. Disponível em: <http://www.scielo.br/scielo.php?script=sci_arttext\&pid=S0104-07072012000100018>.

Acesso em: 11 abr. 2017.

CAMARANO, A.A; KANSO,S; FERNANDES, D; Brasil envelhecer antes e pós-PNI. In: ALCÂNTARA, A.0; CAMARANO, A.A; GIACOMIN, K.C. (Org). Políticas Nacional do Idoso: velhas e novas questões. Rio de Janeiro: IPEA, 2016, p. 63-103.

CARVALHO, J.A.M.; RODRÍGUEZ-WONG,L.L. Transição da estrutura etária brasileira.Cad. Saúde Pública, Rio de Janeiro, v.24, n.3, p.597-605, mar 2008.

FECHINE, B.R.A; TROMPIERI, N; 0 processo de envelhecimento: as principais alterações que acontecem com o idoso com o passar dos anos. Inter Science Place, ed.20, v 1, n. 07, p. 106-132 Ceará, Jan/Mar 2012. Disponível em:

http://ftp.interscienceplace.org/isp/index.php/isp/article/view/196/194

Acesso em 05 jul. 2017.

FROEHLICH, J. M. et. al. Êxodo seletivo, masculinização e envelhecimento da população rural na região central do RS. Ciência Rural. Santa Maria, v. 41, n. 9, p. 1674-1680, 2011. Disponível em: <www.redalyc.org/html/331/33119939031/>. Acesso em: 12 jul. 2017.

IBGE - Instituto Brasileiro de Geografia e Estatística.Nível territorial - Distrito, 2010. Disponível em: <https://sidra.ibge.gov.br/Acervo?nivel=10\&unidade=431680807\#/S/Q>. Acesso em: 07 jul. 2017. MISSIO, Martha; PORTELLA, Marilene. Atenção aos idosos rurais no contexto da família: um desafio para a equipe do programa saúde da família. Boletim da Saúde, Porto Alegre, v. 17, n. 2, p. 25-36, 2003.

QUADROS, V.J; MAIA, A.G. Estrutura Sócio Ocupacional no Brasil.Revista de Economia Contemporânea, Rio de Janeiro, v. 14, n. 3, p. 443-468, set/dez. 2010. Disponível em: <http://www.scielo.br/scielo.php?script=sci_arttext\&pid=S141598482010000300001\&lng=en\&nr $\mathrm{m}=$ iso\&tlng=pt>. Acesso em: 12 abr. 2017.

SAKAMOTO, Camila Strobl. Mudanças na composição das famílias e impactos na distribuição de rendimentos: um comparativo entre áreas rurais e urbanas no Brasil.2013. $138 \mathrm{f}$. Dissertação (Programa de Pós-graduação em desenvolvimento econômico)- Universidade Estadual de Campinas, Campinas, 2013. Disponível em: <http://repositorio.unicamp.br/bitstream/REPOSIP/286500/1/Sakamoto,\%20Camila \%20Strobl_M.pdf>. Acesso em: 22 abr. 2017.

SAKAMOTO, C.; MAIA, A. G. Os impactos das mudanças na estrutura das famílias sobre a distribuição de renda: uma comparação entre áreas urbanas e rurais no Brasil. Belém.: 2013. 51 Congresso da 
Sociedade Brasileira de Economia, Administração e Sociologia Rural. Disponível em: http://www.bibliotecadigital.unicamp.br/document/?code=000927432. Acesso em: 12 de abril.2017.

TAVARES, Darlene et. al. Perfil sociodemográfico, capacidade funcional e qualidade devida de homens idosos residentes na zona rural. Revista de Enfermagem e Atenção à Saúde, v. 1, n. 1, p. 1629, 2012. Disponível em:

<http://seer.uftm.edu.br/revistaeletronica/index.php/enfer/article/view/313>.Acesso em: 11 abril, 2017. 